\title{
Communication Architecture of a system to find persons inside ATLAS
}

\author{
C. Cardeira \\ IDMEC/IST, \\ Technical University of Lisbon \\ 1049-001 Lisboa - Portugal \\ carlos.cardeira@ist.utl.pt
}

\author{
O. Beltramello, H. Burckhart, S. Franz \\ CERN
}

CH-1211 Genève 23 - Switzerland \{Olga.Beltramelo, Helfried.Burckhart, Sebastien.Franz@cern.ch\}@cern.ch
G. Benincasa ${ }^{1}$, A. Maio ${ }^{1,2}$, ${ }^{2}$ CFNUL, ${ }^{2}$ FCUL , ${ }^{1,2}$ LIP 1000-149 Lisboa - Portugal Gianpaolo.Benincasa@cern.ch, amelia@lip.pt

\begin{abstract}
ATLAS is a high energy particle detector of unprecedented size and complexity that is under construction at CERN, in the Geneva area. The detector is housed in a cavern of about $50000 \mathrm{~m} 3$ at $100 \mathrm{~m}$ under ground. During the maintenance periods it is expected that up to 150 people could be present in the cavern at the same time, most of them working inside the intricacies of the detector and completely hidden and invisible from outside. In case of emergency, especially if smoke or fog (leaks of cryogenic fluids) are present, it could be extremely difficult and dangerously long for a rescue team to locate every person who could be in danger. Under these circumstances, a granular system for finding persons is then mandatory. FPIAA (Finding Persons Inside ATLAS Areas) is based on a large number (at the present about 400) of Passive InfraRed sensors, each one detecting the presence of a person in a relatively small volume and distributed to cover the most critical locations in the cavern. The information is collected by a computer system and is sent to the Control Room where the operator can follow on a synoptic screen the movements of the persons in the cavern and inside ATLAS. This paper presents the communication architecture that was used for this project.
\end{abstract}

\section{Introduction}

The most important project under development at CERN is the so called LHC (Large Hadron Collider): inside an underground tunnel of about $27 \mathrm{~km}$, two intense proton beams run in opposed directions and are accelerated at the very high energy of $7 \mathrm{TeV}$. The two beams run parallel each other, except in four positions where they collide, producing new particles. At each one of the four collision points, there are installed huge and complex particle detectors which permit to investigate the intimate constitution of the matter. The biggest and more complex of these detectors is called ATLAS (Fig. 1). For further information about ATLAS experiment goals see the movie available for download in the address http://atlas.ch/movie/index.html.

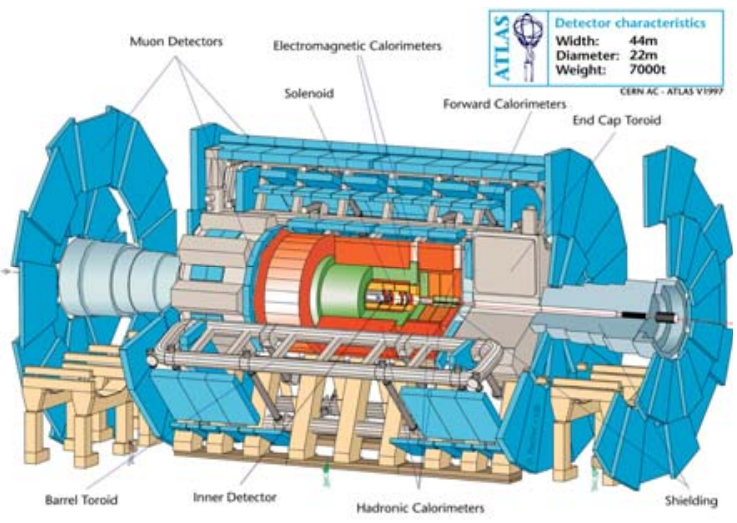

Figure 1. Cut view of the ATLAS detector.

ATLAS is a huge, highly complex physics detector, to a large extent using unconventional and technologically advanced components. ATLAS is composed of various concentric, independent subdetectors, each one based on a different principle of detection and each one performing a specific type of measurement [Atlas 1994]. In case of necessity (maintenance, repair...) the movable parts of the detector can be extracted using appropriate tools, so providing access inside ATLAS.

The detection principles used in ATLAS produce several kinds of risks for persons. Amongst others we recall the large quantities of cryogenic fluids (liquid Argon, liquid Nitrogen and liquid Helium), suffocating and explosive gases, fire, high voltages, thousands of Laser beams for alignment and calibration, ionizing radiations, magnetic fields [Kate 1999], etc. Despite the meticulous care that has been put in the design and implementation of the alarms and safety systems, a mishap is always possible and a particular attention must then be devoted to the problem of the rapidity of intervention [2].

For economical reasons, the regions around ATLAS in the cavern, are extremely exiguous and are largely 
filled with equipment and services. There are gangways, stairs, cranes access etc., where people could be for maintenance and repair purposes. During the maintenance periods, tens (up to more than one hundred) of specialists will enter into the cavern for verifications, checking or repairing activities.

Unlike other big Experiments, it will be possible and necessary to penetrate inside ATLAS hidden regions, completely not visible from outside and where crawling is a more appropriate term than walking. Moreover the hidden paths inside these regions can be several tens of meters long, so a person could have to be working very far from his entering position. In [2] we show some of the possible positions where persons could be.

A reliable access system exists that provides at any moment precise information on the total number of persons present in the cavern: however no information exists on where these persons are located. In case of urgency (smoke, gas leakage, cryogenic leakage, etc...) an evacuation signal will urge people to leave the cavern: however if the number of exiting people does not match with the number of present persons, it would be extremely difficult and dangerously long for the rescue team to find a person in this maze. It is then essential to know at each moment where the persons are inside ATLAS and especially inside the most dangerous regions in the cavern.

This information will be provided by the FPIAA system.

\section{Acquisition system}

The major constraint for a FPIAA system is certainly represented by the presence of large quantities of metallic material which could prevent the propagation of electromagnetic signals. Apart from the feet and the main support structures and rails, which are made of stainless steel, most of the ATLAS parts are made of various metals. During the design phase of the project, one of the major specifications was the possibility to identify the persons by name ("who" is in that area). Various alternatives were investigated: most of them used a transmitter-receiver technique that resulted or too expensive, or not compatible with the environment constraints. So the idea of identification by name was abandoned and we limited the requirement to a simpler person detection ("a" person is in that area). This restriction is not fundamental from the point of view of the security [2].

After a study of possible solutions for detecting persons [2], the previous considerations oriented our choice on two types of detectors: the Ultrasonic or Passive Infrared (PIR) devices. Due to magnetic and irradiation tests Ultrasonic Sensors were abandoned and the final solution is based on PIR sensors.
The regions concerned have then been divided in small cells, each one provided with a detector which will monitor the presence of a person in the specific cell. The signals coming from all the cells will be treated by a computer and a synoptic will be presented in Control Room where coloured cells will indicate, on an ATLAS map, the positions of the various persons.

However, this system presents an important drawback: both the PIR and Ultrasonic detectors can only detect moving persons. In the case a person is unconscious or, worst, is dead, the signal from the cell concerned will simply disappear. This problem might be solved by using models of the system and building observers for its state: most of the cells are contiguous each other, at least in the more dangerous regions, so if a person disappears from a cell without re-appearing in a contiguous cell, an alarm can be produced.

\subsection{Sensors}

The sensors chosen [2] were PIR sensors shown in figure 2. These are low cost sensors that, like most of the other brands we tested, have to be modified to cope with the irradiation level and the strong magnetic filed existing in ATLAS [2].

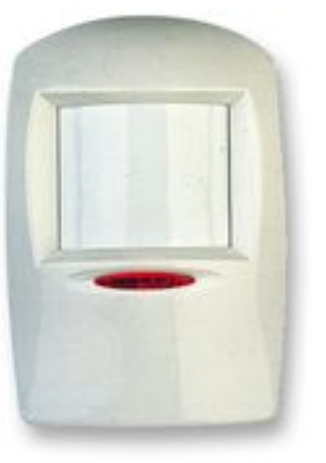

\section{Figure 2. Passive Infra Red (PIR) sensor.}

The position of all the sensors to cover ATLAS areas is known. Between 300 and 400 sensors are necessary to cover all the regions were persons can be.

Such large scale acquisition system needs acquisition cards to read the sensors and a fieldbus to convey the information to the control room.

This function is made by the ATLAS DCS system [3].

\subsection{Signal acquisition and conditioning}

The ELMB (Embedded Local Monitor Board) is the general purposed card developed at CERN for digital and analogue signal acquisition and conditioning [6]. It is a low cost board, able to read 64 analog inputs and 
connects to the industry standard CANbus. CANopen has been implemented as high-level communication protocol.

Its main features are:

- Full CAN controller interface with PCA82C250

- 18 digital bi-directional I/O lines.

- 8 digital output lines.

-8-channel 10-bit ADC

- 8 digital input lines or 8 analog inputs for the ADC

- 6 bipolar or unipolar input ranges from $25 \mathrm{mV}$ to $5 \mathrm{~V}$

- 64 channel multiplexer

The ELMB is standard at CERN and is able to work under the irradiations and strong magnetic field of ATLAS.

Some pictures of the ELMB are presented in figure 3. As we may see in the figure, the ELMB itself is a small size card that is plugged in a expansion board to give access to all buffers, plugs and sockets for all the $\mathrm{I} / \mathrm{O}$ and analog ports available.

FPIAA only uses ELMB digital inputs, two per sensor, one for the sensor signal and the other to check if the sensor is powered. Other available inputs are used for other DCS needs.
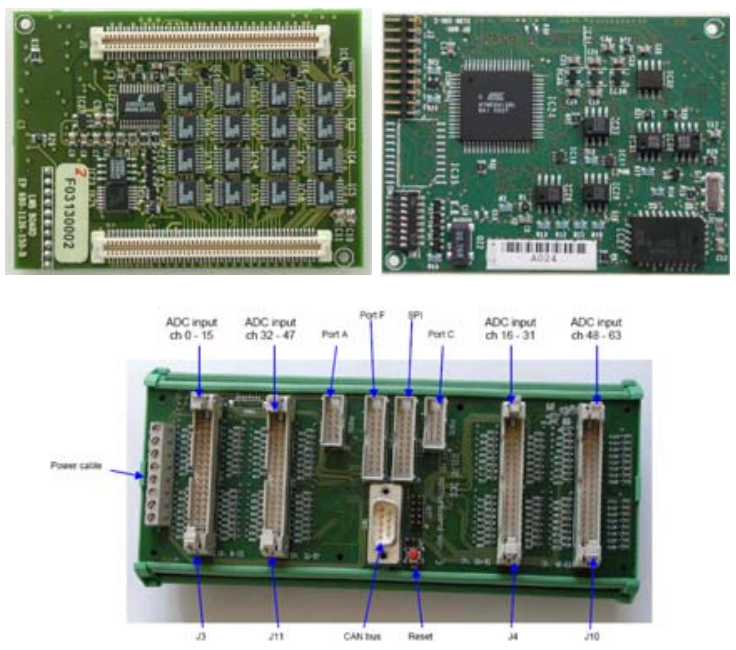

Figure 3. ELMB card

The ELMB possesses its own microcontroller as well as a microcontroller to interface to the CAN bus. The block diagram is presented in figure 4 [7].

\subsection{Fieldbus}

In the 90's, there was a period of discussion to decide which fieldbus should be chosen at CERN. Several proposals were made, manufacturers made proposals scientists made requirements and several meetings and workshops were organized. The conclusion was that CERN recommended the use of the following fieldbuses [1]: CAN bus, PROFIBUS and WORLDFIP.

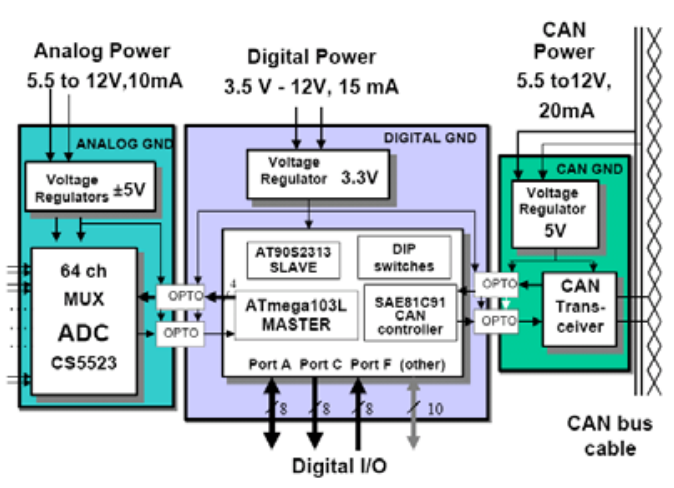

Figure 4. ELMB block diagram

In ATLAS DCS the choice was CAN bus and hence FPIAA was adapted to the use of this bus. A prior proofof-the-concept FPIAA prototype was made with another fieldbus but for the final installation it was necessary to adapt the software and hardware to $\mathrm{CAN}$, as the advantages of using a standard implementation were significant.

The sensors were them connected to an ELMB which is the standard solution at CERN and is developed for the CAN bus.

A study was made to decide were to pass the cables. Moreover, there was a need to calculate the power cables size to cope with admissible attenuations of the power and signal that are transmitted along the CAN cable. For this study a simulator was developed [5]. In the simulator it is possible to enter the different cables length, the number of ELMBs, the sensor number and the power consumption of each sensor. The simulator validates, or not, the installation. The cables there were installed in sector 13 pilot site were dimensioned according to this study.

\section{Software Architecture}

The ELMBs connected to the CAN bus which goes to a control room where an industrial PC with a CAN Kvaser card collects all the information.

\subsection{CAN open}

The PC access the CAN bus data using the CAN Open stack of communications.

\subsection{OPC Server}


The same PC runs an OPC server which gives access to the data for the several installed clients.

\subsection{OPC Client}

The standard OPC client at CERN is the SCADA software PVSS.

However, for FPIAA application we felt that a programming language would be more suited for handling the estimation system of persons presence based on sensor actuation. However, the SCADA system is well suited for monitoring the state of sensors as well as maintaining logs of data.

To interface the application with the OPC server we developed an OPC client.

The OPC client was developed using Measurement Studio package from National Instruments. In the National site we are able to found an open source that uses DCOM services to browse the network for OPC servers and list the available data. Using this software we were able to browse the local host, find a local OPC server and after some minor modifications on the source, show the state of one sensor, as shown in figure 4 .
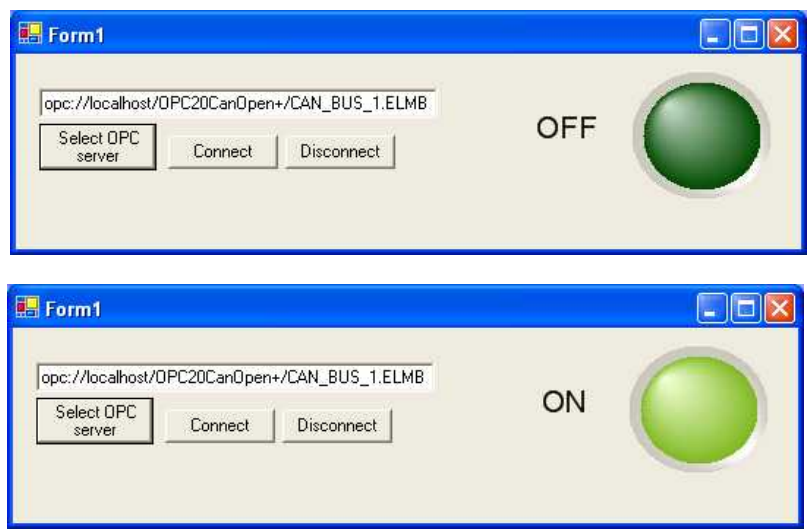

Figure 4. OPC client test

The OPC client made uses the DCOM services but recent firewalls and security protections are turning it more difficult to run. Often, a simple upgrade of the operating systems creates large troubles for the programs to continue working. This is a general issue of all the technologies but in this case we felt it in particular in such a way that it is not decided if in the final implementation we will use our own client or a standard PVSS client to access the data and make our program run on top of a PVSS client communication with it with local active $\mathrm{X}$ commands instead of remote OPC calls.

\section{Experimental site}

The experimental site was installed in the ATLAS pit, on the first levels.

Figure 5 shows the ATLAS detector. The pilot site was installed in the levels indicated in the figure.

Along each level 3 to 4 sensors were placed and connected to the nearest ELMBs.

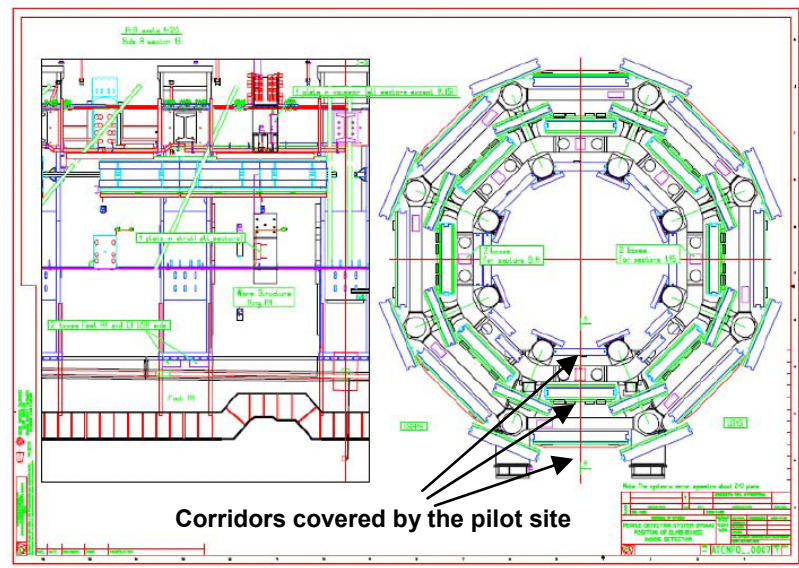

Figure 5. Pilot site

\section{Results}

To test the experiment the following procedure was followed:

The connection of the sensors to the ELMB, was checked with local tests.

The connections between the ELMB and the CAN cable was checked with a laptop with a CAN PCMCIA adapter to be able to read the sensor values with a traffic monitor.

The CAN cable connections between the ELMB and the Control Room (which is 100-200 meters far away in cable length) was checked with the traffic monitor running at the Control Room PC.

When all the systems were checked and operating correctly from the hardware point of view, we were able to make a test with a testing PVSS program to check the good operation of the entire system.

A small PVSS client was then developed to test the entire DCS architecture for FPIAA.

Figure 6 shows a screencapture of the PVSS window showing the states of the sensors changing accordingly to the movement of the persons in the areas covered by the sensors. 


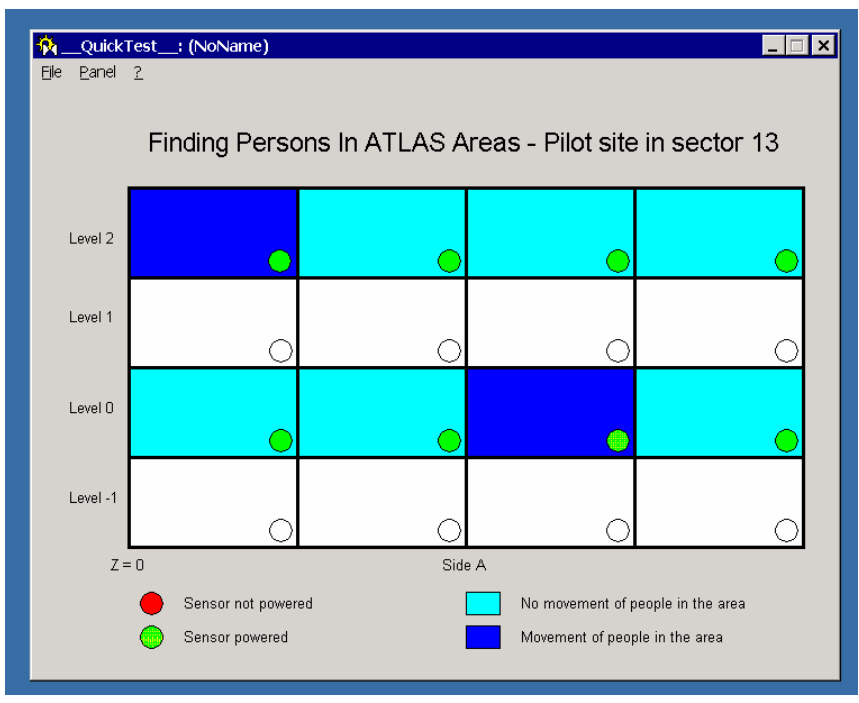

Figure 6. Remote view of the sensors state

\section{Conclusions}

In this paper we presented the communication architecture of an original persons detection system in which the people position is detected without need of badges or other sort of personal identifiers.

The system is large in size and in the number of distributed information to be acquired.

The system is supported by a CAN network with intelligent nodes (ELMB) which acquire the information from the sensors which is collected by a remote CAN OPEN OPC server.

The data is then access by a PVSS client that can be used to generate HMI to operators as well as perform logging of the sensors state.

Further work will include the development of an intelligent system to estimate the persons positions along the areas. This situation is a problem of tracking with occlusions was two persons in one area are not distinguished of one person in the same area [4].

Moreover, particular situations will generate alarms as it might be possible to detect vanishings if some abnormal situations occur like, for instance, an absence of movement in a certain sensor without movement being detected by the sensors in the neighborhood areas.

\section{References}

[1] G. Baribaud, R. Barillere, A. Bland, D. Brahy, H. Burckhart,R. Brun, E. Carlier, W. Heubers, L. Jirden, F. Perriollat, D. Swoboda, "Recommendations for the Use of Fieldbuses at CERN in the LHC Era", ICALEPCS'97, November 3-7, Beijing, 1997.

[2] Gianpaolo Benincasa, Carlos Cardeira, David Claudino, Amelia Maio, "FPIAA -Find Persons Inside Atlas Areas: a system for finding and rescuing persons in a very large physics experiment", in SLAC-WP-049 the 5th International High Energy Physics Technical Safety Forum Workshop, Menlo Park, California, April 11-15, 2005.

[3] Burkhart, H. , "Detector Control Systems", Fourth Workshop on Electronics for LHC Experiments", Rome, Italy, 21 to 25 September, 1998.

[4] Cucchiara, R.; Grana, C.; Tardini, G.; Vezzani, R.; "Probabilistic people tracking for occlusion handling", Proceedings of ICPR 2004, the 17th International Conference on Pattern Recognition, Volume 1, 23-26 Aug. 2004 Page(s): 132 - 135.

[5] S. Franz. H. Buckhart, "Simulator verify voltage over long CAN cables", available online at http://atlas.web.cern.ch/Atlas/GROUPS/DAQTRIG/DCS /ELMB/SIMULATION/simulation.html.

[6] Hallgren B., Baehler P., Burckhart H.J., Filimonov V., Hallewell G., Karlov A., Kersten S., Kind P., Merkel M., Poggioli L., Raybov Y., Samoilov V., Sandaker H., Takai H., "A Low Cost I/O Concentrator Using the CAN Fieldbus", ICALEPS 99 conference, Trieste, Italy, 4 - 8 October 1999

[7] Hallgren, B; Boterenbrood, H.; Burckhart, H.; Kvedalen, H. "The Embedded Local Monitor Board (ELMB) in the LHC Front-end I/O Control System", 7th Workshop on Electronics for LHC Experiments", Stockholm, Sweden, 10 to 14 September, 2001. 